\title{
Évaluation des teneurs sériques en sodium et en calcium et de leurs potentialités de transfert dans le complexe maternello-placento-fœtal (CMPF) des chèvres en zone tropicale.
}

\author{
Ngoie K ${ }^{1}$, Kasereka S.B ${ }^{2^{*}}$, Kaluendi C.M. ${ }^{3}$ Ndibualonji B.B., Kaputo M${ }^{1}$. \\ ${ }^{1}$ Service de physiologie et physiopathologie, Faculté de Médecine Vétérinaire, Université de Lubumbashi, B.P.1825, \\ Lubumbashi, R.D. Congo. \\ ${ }^{2}$ Service d'Anatomo-pathologique, Autopsie et Pathologie aviaire Faculté de Médecine Vétérinaire, Université de \\ Lubumbashi, B.P.1825, Lubumbashi, R.D. Congo. \\ ${ }^{3}$ Service de Biochimie normale et Pathologique, Faculté de Médecine Vétérinaire, Université de Lubumbashi, \\ B.P.1825, Lubumbashi, R.D. Congo. \\ *Auteur chargé de la correspondance : E-mail : bourg.2013@gmail.com \\ Original submitted in on $9^{\text {th }}$ October 2016. Published online at www.m.elewa.org on 31st December 2016 \\ http://dx.doi.org/10.4314/jab.v108i1.3
}

\section{RÉSUMÉ}

L'objectif : le but de cette étude était d'évaluer les teneurs sériques en sodium et en calcium ainsi que leurs intensités de transfert dans le CMPF au cours de la gestation chez les chèvres élevées à Lubumbashi à zone tropicale.

Méthodologie et résultats: Cette recherche a été menée sur 45 sérums dont 15 provenant de chèvres gestantes $(\mathrm{Gg}), 15$ de leurs placentas $(\mathrm{Pg})$ et 15 de leurs fœtus $(\mathrm{Fg})$ abattues dans les tueries du marché mzee Kabila à Lubumbashi. Les teneurs sodiques et calciques ont été obtenues au spectrophotomètre par dosage colorimétrique. Nous avons obtenu dans l'ensemble du CMPF 489,2 $\pm 335,8 \mathrm{mmol} / \mathrm{l}(100 \%)$ pour le sodium et de $4,1 \pm 1,1 \mathrm{mmol} / /$ pour le calcium. Les teneurs sodiques des chèvres gestantes, placentas et fœetus étaient respectivement de 121,0 $\pm 69,2 \mathrm{mmol} / \mathrm{l}(24,7 \%), 197,0 \pm 225,8 \mathrm{mmol} / \mathrm{l}(40,3 \%)$ et $171,2 \pm 172,8$ $\mathrm{mmol} / \mathrm{l}(35 \%)$. L'évaluation statistique a révélé que les natrémies placentaire et fœtale étaient significativement plus élevées que celles des femelles gestantes. Les calcémies moyennes étaient de $1,33 \pm 0,5 \mathrm{mmol} / \mathrm{l}(32,2 \%), 1.26 \pm 0,4 \mathrm{mmol} / \mathrm{l}(30.5 \%)$ et $1,54 \pm 0,7 \mathrm{mmol} / /(37,3 \%)$, respectivement chez les chèvres gestantes, les placentas et les fœtus. L'évaluation statistique a révélé des différences significatives entre la calcémie maternelle et fœtale et entre la calcémie placentaire et fœtale.

Conclusion et application des résultats : Les résultats de cette recherche ont permis de comprendre qu'à climat tropical de Lubumbashi, la chèvre gestante peut être menacée d'hyponatrémie, d'hypocalcémie et que la supplémentassions s'avère nécessaire.

Mots-clés : Natrémie, calcémie, potentialités de transfert, complexe maternello-placento-fœtal, chèvres en zone tropicale. 


\title{
Ngoie et al., J. Appl. Biosci. 2016 Évaluation des teneurs sériques en sodium et en calcium et de leurs
} potentialités de transfert dans le complexe maternello-placento-fœetal (CMPF) des chèvres en zone tropicale.

\section{Evaluation of sodium and calcium serum content and their transfer potentials in the maternello- placento-fetal complex (cmpf) of goats in the tropical zone}

\begin{abstract}
.
Objective: The aim of this study was to evaluate serum sodium and calcium levels and their transfer rates in MPFC during gestation in goats raised in Lubumbashi in tropical areas.

Methods and results: This research was carried out on 45 serums of which 15 were from pregnant goats $(\mathrm{Gg}), 15$ from their placentas $(\mathrm{Pg})$ and 15 from their fetuses $(\mathrm{Fg})$ slaughtered in the mzee Kabila market in Lubumbashi. The sodium and calcium contents were obtained by spectrophotometer by colorimetric determination. We obtained $489.2 \pm 335.8 \mathrm{mmol} / \mathrm{I}(100 \%)$ for sodium and $4.1 \pm 1.1 \mathrm{mmol} /$ I for calcium in the CMPF as a whole. The sodium levels of pregnant goats, placentas and fetuses were respectively 121.0 $\pm 69.2 \mathrm{mmol} / \mathrm{I}(24.7 \%), 197.0 \pm 225.8 \mathrm{mmol} / \mathrm{I}(40.3 \%)$ and $171.2 \pm 172.8 \mathrm{mmol} / \mathrm{I}(35 \%)$. Statistical evaluation revealed that placental and fetal serum natremia were significantly higher than those of pregnant females. Mean calcemia were $1.33 \pm 0.5 \mathrm{mmol} / \mathrm{I}(32.2 \%), 1.26 \pm 0.4 \mathrm{mmol} / \mathrm{I}(30.5 \%)$ and $1.54 \pm 0.7$ $\mathrm{mmol} / \mathrm{I}(37.3 \%)$, Respectively in pregnant goats, placentas and fetuses. Statistical evaluation revealed significant differences between maternal and fetal calcium and between placental and fetal calcium.

Conclusion and application of results: The results of this research made it possible to understand that in the tropical climate of Lubumbashi, the pregnant goat may be threatened with hyponatraemia, hypocalcemia and that supplementation is necessary.
\end{abstract}

Keywords : Natraemia, calcemia, transfer potential, maternal-placento-fetal complex, goats in tropical areas.

\section{INTRODUCTION.}

Le sodium et le calcium sont des cations majeurs du milieu extracellulaire. Le calcium est parathormone et calcitonino-dépendant (Pineda et al., 2003), tandis que le sodium est aldostéronodépendant (Boudon et al.,2013), Dans l'organisme, ces deux cations sont soit associés dans certains processus fonctionnels (excitabilités neuromusculaires) soit agissent isolement dans d'autres processus, la rétention hydrique pour le sodium, la croissance osseuse pour le calcium (Suttle, 2010). Le besoin en sodium et en calcium peuvent dépendre de l'état physiologique, principalement de la gestation et de la lactation (Gilles et al., 2006). Au cours de la gestation, la mère reste la principale pourvoyeuse de la plus part des molécules dont le sodium et calcium. Chez la mère ces cations peuvent être d'origine endogène à partir des réserves tissulaires, alors que pour le placenta et le fœtus, ils sont à 100\% d'origine maternelle grâce au transfert maternellofœtal (Bell et al., 2005 ; Tabatabaei, 2012 ; Hater et al., 2015). Ce transfert maternello-fœtal de différentes molécules dépend des potentialités maternelles, des besoins fœtaux et de l'efficacité de système de transport: gradient de concentration, système d'échangeurs, transport actif et passif, intervention de protéines de transports (Bell et al., 2005). Les transferts globaux de sodium et calcium ne correspondent pas aux teneurs dosables, mais ils sont très élevé compte tenu de teneurs non dosables séquestrées au niveau placentaire et fœtal, dans les tissus placento-fœtaux ainsi que dans les liquides fœtaux (amniotique et allantoïde) (Tabatabaei, 2012). La natrémie, la calcémie est évaluée différemment par certains auteurs chez la chèvre gestante (Kaneko et Cornelius, 1970 ; Ouedraogo et al., 2008 ; Hater et al.,2015), chez le fœtus pour le calcium (Farell, 1984). Néanmoins, la littérature reste muette pour la natrémie et calcémie placentaire. C'est pourquoi cette recherche vise à évaluer non seulement les teneurs sodiques, calciques dans le sang de chèvres gestantes, mais aussi de leurs placentas, de leurs fœtus et analysés les potentialités de transfert de ces minéraux dans le CMPF étant donné que la supplementations minérale en élevage caprin de Lubumbashi est une pratique rare. 


\section{Ngoie et al., J. Appl. Biosci. 2016 Évaluation des teneurs sériques en sodium et en calcium et de leurs}

potentialités de transfert dans le complexe maternello-placento-fœetal (CMPF) des chèvres en zone tropicale.

\section{MATERIELS ET METHODOLOGY}

Milieu : Cette recherche a été effectuée à Lubumbashi dans les tueries de petits animaux au marché Mzee KABILA pour le prélèvement et au laboratoire de biochimie de la Faculté de Médicine Vétérinaire de l'Université de Lubumbashi pour le dosage. La ville de Lubumbashi est géographiquement située à $11^{\circ} 40^{\prime}$ de latitude sud et à $27^{\circ} 28^{\prime}$ de longitude Est, à 1268 mètres d'altitude.

Animaux : Pour cette recherche, nous nous sommes servis des 45 sérums dont 15 issus des chèvres gestantes, 15 de leurs placentas et 15 de leurs fœtus provenant des élevages péri-urbain de Lubumbashi, nourris exclusivement sur pâturage naturel et ne recevant pas aucune supplémentassions minérale.

Matériels : Pour le prélèvement du sang, le couteau, les seringues, les tubes à essai sans anticoagulant, les portoirs, le marqueur, l'ouate et les gants étaient utilisés. La centrifugeuse, les seringues, les tubes à essai, les pipettes graduées et le réfrigérateur nous ont permis d'obtenir et conserver les sérums. Tandis que le spectrophotomètre, les herlens meyer, les pipettes automatiques, les embouts en caoutchouc, l'ouate, les cuvettes optiques, les porte-tubes, les tubes à essai, le kit de sodium et le kit de calcium de la maison Cypress diagnostic Belgique ont constitué les matériels de dosage.

Méthodes: Pour le prélèvement, 5 à10 $\mathrm{ml}$ des échantillons de sang étaient obtenus des chèvres gestantes lors de l'égorgement. Le sang placentaire ( \pm $5 \mathrm{ml}$ ) était obtenu à la suite de l'incision dans la jonction placenta-ombilicale tandis que le sang fœtal $(6 \mathrm{ml})$ était recueilli en incisant les deux veines jugulaires. Puis, 3 à $5 \mathrm{ml}$ de sérum étaient recueillis de chaque échantillon après centrifugation et étaient conservés au réfrigérateur jusqu'au moment des analyses qui se faisaient le même jour. Le dosage de sodium et de calcium ont été réalisés par le test colorimétrique selon les recommandations spécifiques de la maison Cypress Diagnostique Belgique. Pour l'analyse statistique, mous avons procédé aux calculs des moyennes, écart-types, variances et estimations selon les méthodes classiques. Le test $\mathrm{T}$ des student nous a permis de comparer les moyennes deux à deux. Les différences ont été considérées significatives au seuil de $5 \%$.

\section{RESULTATS}

Les résultats des concentrations sodiques et calciques de chaque CMPF sont regroupés dans les tableaux 1, 2 et 3 .

Tableau 1: Teneurs sodiques (mmol/l) par CMPF des chèvres gestantes $(\mathrm{Gg})$; leurs placentas (Pg et leurs fœtus $(\mathrm{Fg})$.

\begin{tabular}{lllll}
\hline $\mathbf{N}^{\mathbf{0}}$ & $\mathbf{C} \mathrm{CMPF} / \mathbf{1 0 0} \%$ & $\mathbf{G g} / \%$ & $\mathbf{P g} / \%$ & $\mathbf{F g} / \%$ \\
\hline 1 & 464,92 & $113,27 / 24,4$ & $21,85 / 4,7$ & $329,8 / 70,9$ \\
2 & 198,67 & $37,74 / 19$ & $143,08 / 72$ & $17,87 / 9$ \\
3 & 361,66 & $69,55 / 19,2$ & $216,61 / 59,9$ & $75,5 / 21$ \\
4 & 319 & $160,9 / 50,4$ & $119,23 / 37,4$ & $38,89 / 12,2$ \\
5 & 420,2 & $123,21 / 29,3$ & $101,79 / 24,2$ & $195,2 / 46,5$ \\
6 & 411,36 & $156,99 / 38$ & $69,55 / 17$ & $184,82 / 45$ \\
7 & 1456,87 & $35,76 / 2,5$ & $737,49 / 50,6$ & $683,62 / 46,9$ \\
8 & 169,9 & $32,63 / 19,2$ & $51,65 / 30,4$ & $85,62 / 50,4$ \\
9 & 361,67 & $119,23 / 33$ & $220,59 / 61$ & $21,85 / 6$ \\
10 & 432,24 & $109,29 / 25,3$ & $47,67 / 11$ & $275,28 / 63,7$ \\
11 & 482,82 & $83,46 / 17,3$ & $117,18 / 24,3$ & $282,18 / 58,4$ \\
12 & 325,87 & $125,18 / 38,4$ & $135,12 / 41,5$ & $65,57 / 20,1$ \\
13 & 464,08 & $262,24 / 56,5$ & $113,27 / 24,4$ & $88,57 / 19,1$ \\
14 & 1075,1 & $254,35 / 23,7$ & $735,31 / 68,4$ & $85,44 / 7,9$ \\
15 & 393,42 & $131,14 / 33,3$ & $125,18 / 31,82$ & $137,1 / 35$ \\
\hline $\mathbf{M} \mathbf{+ S}$ & $489,2 \pm 335,8$ & $121,0 \pm 69,2$ & $197,0 \pm 225,5$ & $171,2 \pm 172,8$ \\
$\%$ & 100 & 24,7 & 40,3 & 35 \\
\end{tabular}

Légende : $\Sigma$ CMPF : la somme du complexe maternello-placento-fœtal, M: moyenne, S : écart-types. 


\section{Ngoie et al., J. Appl. Biosci. 2016 Évaluation des teneurs sériques en sodium et en calcium et de leurs}

potentialités de transfert dans le complexe maternello-placento-fœetal (CMPF) des chèvres en zone tropicale.

Ce tableau révèle que pour la natrémie globale de $489,2 \pm 335,8 \mathrm{mmol} / \mathrm{l}(100 \%)$, les natrémies maternelle, placentaire et fœtale sont respectivement de $24,7 \%$, $40,3 \%$ et $35 \%$. les calculs statistiques ont révélé que la natrémie maternelle est faible par rapport aux natrémies placentaire et fœtale, alors qu'il n'y a pas de différence significative entre la natrémie placentaire et la natrémie fœtale.

Tableau 2 : Teneurs calciques (mmol//) par CMPF des chèvres gestantes, leurs placentas et leurs fœtus

\begin{tabular}{lllll}
\hline $\mathbf{N}^{\circ}$ & $\mathbf{C}$ CMPF/100\% & $\mathbf{G g} / \%$ & $\mathbf{P g} / \%$ & $\mathbf{F g} / \%$ \\
\hline 1 & 4,45 & $1,83 / 41,1$ & $1,03 / 23,1$ & $1,59 / 35,7$ \\
2 & 5,77 & $1,87 / 32,4$ & $0,95 / 16,5$ & $2,95 / 51,1$ \\
3 & 3,72 & $1,20 / 32,2$ & $1,42 / 38,2$ & $1,1 / 29,6$ \\
4 & 3,58 & $0,85 / 23,7$ & $1,51 / 42,2$ & $1,22 / 34,1$ \\
5 & 3,44 & $1,26 / 36,6$ & $1,01 / 29,4$ & $1,7 / 34$ \\
6 & 3,35 & $1,20 / 35,8$ & $0,93 / 27,8$ & $1,22 / 36,4$ \\
7 & 4,24 & $0,86 / 20,3$ & $2,48 / 58,5$ & $0,90 / 21,2$ \\
8 & 3,67 & $1,12 / 30,5$ & $0,95 / 26,4$ & $1,58 / 43,1$ \\
9 & 3,26 & $1,18 / 36,2$ & $1,14 / 35$ & $0,95 / 28,8$ \\
10 & 3,32 & $1,16 / 35$ & $1,19 / 35,8$ & $0,97 / 29,2$ \\
11 & 1,91 & $0,09 / 5$ & $0,91 / 47,6$ & $0,91 / 47,6$ \\
12 & 4,88 & $1,30 / 26,6$ & $1,14 / 23,4$ & $2,44 / 50$ \\
13 & 4,67 & $2,25 / 48,2$ & $1,18 / 25,3$ & $1,24 / 26,5$ \\
14 & 5,6 & $1,95 / 35$ & $1,57 / 28$ & $2,08 / 37,1$ \\
15 & 6,02 & $1,79 / 29,7$ & $1,46 / 24,3$ & $2,77 / 46$ \\
\hline $\mathbf{M} \mathbf{+ S}$ & $4,1 \pm 1,1$ & $1,33 \pm 0,5$ & $1,26 \pm 0,4$ & $1,54 \pm 0,7$ \\
$\mathbf{\%}$ & 100 & 32,2 & 30,5 & 37,3 \\
\hline
\end{tabular}

Ce tableau laisse observer que pour une calcémie globale de $4,1 \pm 1,1 \mathrm{mmol} / /(100 \%)$ dans le CMPF, les teneurs calciques des chèvres gestantes, placentas et fœtus correspondent respectivement à $32,2 \%, 30,5 \%$ et
$37,3 \%$. L'évaluation statistique montre que la calcémie fœtale est supérieure aux calcémies maternelles et placentaires.

Tableau 3 : Valeurs moyennes ( $\mathrm{mmol} / /)$ de la natrémie et de la calcémie dans le CMPF des chèvres gestantes, leurs placentas et leurs fœetus.

\begin{tabular}{lcccc}
\hline & CMPF & Gg & Pg & Fg \\
\hline sodium & $489,2 \pm 335,8$ & $121,0 \pm 69,2$ & $197,0 \pm 225,8$ & $171,2 \pm 172,8$ \\
calcium & $4,1 \pm 1,1$ & $1,33 \pm 0,5$ & $1,26 \pm 0,4$ & $1,54 \pm 0,7$ \\
\hline
\end{tabular}

Ce tableau montre que la natrémie est plus élevée au niveau placentaire mais faible au niveau maternel, alors que la calcémie est plus concentrée au niveau fœtal.

\section{DISCUSSION}

Cette discussion porte sur les teneurs sodiques et calciques globales dans le CMPF, dans les différents compartiments (chèvre gestantes, placentas, fœtus) et l'analyse de tendance de transfert maternello-placentofœtal en fonction des spéculations fonctionnelles.

Natrémie : Pour une natrémie globale moyenne de $489,2 \pm 333,8 \mathrm{mmol} / \mathrm{l}(100 \%)$ dans le CMPF, les natrémies maternelle, placentaire et fœtale sont respectivement de $121,0 \pm 69,2 \mathrm{mmol} / \mathrm{l}(24,7 \%)$, $197,0 \pm 225,8 \mathrm{mmol} / \mathrm{l}(40,3 \%)$ et $171,2 \pm 172,8 \mathrm{mmol} / \mathrm{l}$ (35\%). La comparaison statistique des natrémies moyennes maternelle, placentaire et fœtale a révélé que la natrémie maternelle est significativement plus basse que les natrémies placentaire et fœtale alors qu'il n'existe pas de différence significative entre les natrémies placentaire et fœtale. Ces résultats montrent que le sodium est plus concentré au niveau placentaire et fœtal et que la mère, bien qu'elle soit l'unique source sodique, ne jouerait que le rôle de pompe sodique foulante vers le placenta et vers le fœtus alors que ces 2 derniers, constituent une pompe sodique aspirante au détriment de la mère. En effet, à part le sodium dosé 


\section{Ngoie et al., J. Appl. Biosci. 2016 Évaluation des teneurs sériques en sodium et en calcium et de leurs potentialités de transfert dans le complexe maternello-placento-fœetal (CMPF) des chèvres en zone tropicale.}

dans le sang placentaire et fœetal, une grande partie du sodium maternel est aussi stocké dans les tissus placentaires et fœtaux ainsi que dans les liquides fœtaux (amniotique et allantoïde).II en découle ainsi que les pertes sodiques maternelles sont plus importantes que les teneurs sodiques dosées dans le sang placentaire et fœtal (Hater et al., 2015). Cette faible natrémie maternelle est l'expression d'un transfert intense de sodium vers le placenta et le fœetus, d'une part, et vers les liquides fœtaux, d'autre (Tabatabaei, 2012). En effet, le sodium constitue l'électrolyte osmotique le plus important dont le fœtus a besoin principalement pour maintenir un milieu hydrique nécessaire à sa survie (Suttle, 2010). En outre, pour une quantité suffisante des liquides fœtaux, la mère et placenta doivent transfert intensément le sodium pour prévenir les chocs fœtomaternels (Tabatabaei, 2012). Par ailleurs, en plus du transfert fœto-maternel pour les besoins de gestation, la gestante perd aussi de son sodium vers la salive, le suc digestif, l'urine et la sueur (Hater et al., 2015). La supériorité de la natrémie fœetale par rapport à la natrémie maternelle s'expliquerait par le fait qu'à part ces besoins métaboliques et plastiques, le foetus a grandement besoin de sodium pour d'autres besoins (rétention hydrique, constitution du liquide amniotique et allantoïde) (Tabatabaei, 2012 ; Hater et al., 2015). Pour ces auteurs le taux sodique est très élevé dans les liquides fœtaux au début de la gestation que vers sa fin. Pour le placenta, sa supériorité statistique natrémique $(p<0,05)$ par rapport à la natrémie maternelle et arithmétique $(p>0,05)$ par rapport au fœtus montrerait que le placenta n'est qu'un dépôt et un lieu de passage vers le fœtus, un organe qui régulerait les besoins fœtaux par rapport aux potentialités maternelles (Barone, 2001 ; Ngoie et al., 2016). Le système rénine angiotensine aldostérone en collaboration avec divers systèmes de transporteurs joueraient un rôle régulateur déterminant (Gilles et al., 2006 ; Boudon et al.,2013). Comparée aux natrémies exotiques des zones tempérées, la natrémie de $121,0 \pm 69,2 \mathrm{mmol} / \mathrm{l}$ observée localement chez la chèvre gestante est faible par rapport aux natrémies de 135 $\mathrm{mmol} / \mathrm{l}$ et $141,12 \mathrm{mmol} / \mathrm{l}$ avancée respectivement par Kaneko et Cornelius (1970) et Hater et al. (2015). La faiblesse de la natrémie locale peut être due au manque de compléments alimentaires et éventuellement en une forte déperdition du sodium dans la sueur en climat chaud (Gal et Planchanault, 1993 ; Ouedraogo et al., 2008).
Calcémie : Pour une calcémie globale moyenne du CMPF de $4,1 \pm 1,1 \mathrm{mmol} / \mathrm{l} \quad(100 \%)$, les calcémies maternelle, placentaire et fœtale sont respectivement de $1,33 \pm 0,5 \mathrm{mmol} / \mathrm{l}(32,2 \%), 1,26 \pm 0,4 \mathrm{mmol} / \mathrm{l}(30,5 \%)$ et $1,54 \pm 0,7 \mathrm{mmol} / /(37,3 \%)$. La comparaison statistique des moyennes calciques des chèvres gestantes, de leurs placentas et de leurs fœtus a révélé que la calcémie fœtale est significativement plus élevée que les calcémies maternelle et placentaire alors qu'il n'y a pas de différence significative entre les calcémies maternelle et la calcémie placentaire. Ces résultats montrent que pour le calcium, le fœtus est prioritaire pour assurer principalement ses besoins de croissance et ses besoins métaboliques et que le calcium est rapidement transféré de la mère vers le placenta et du placenta vers le fœtus. Les pertes calciques maternelles au cours de la gestation sont plus importantes que les teneurs dosées dans le sang, une partie du calcium étant séquestrée dans les tissus placentaires et fœtaux plus particulièrement dans les os, dans les molécules et les liquides organiques autre que le sang (Liesegang et al., 2006 ; Hater et al., 2015). L'infériorité du calcium maternel par rapport au calcium fœtal est principalement due aux sollicitations placentaires et surtout plastiques du fœtus. Pour répondre à toute ces sollicitations, la mère recourt en plus des apports alimentaires à des ressources endogènes par la déminéralisation osseuse sous l'action de la parathormone (Payene, 1983 ; Bossard et al., 2009). L'infériorité de la calcémie placentaire par rapport à la calcémie fœtale s'expliquerait par le fait que le placenta n'est qu'un lieu de passage rapide du calcium vers le fœtus. La supériorité de la calcémie fœtale par rapport à la calcémie maternelle est surtout l'expression des besoins plastiques et métaboliques urgent pour répondre aux sollicitations fœtales. En effet, le fœetus a grandement besoin d'un apport très important de calcium pour assurer sa croissance osseuse. En plus des besoins métaboliques et des croissances osseuses, le calcium est aussi transféré dans les liquides fœtaux. En effet, selon Tabatabaei (2012), le transfert de calcium dans les liquides fœtaux augmente avec l'âge de gestation. Dans ce même ordre d'idées Ouedraogo et al. (2008), rapportent que la concentration de la calcémie des chèvres gestantes diminue avec l'avancement de la gestation. En comparant la calcémie obtenue localement qui est de $1,33 \mathrm{mmol} / \mathrm{l}$ pour les chèvres gestantes, de 1,26 $\mathrm{mmol} / / \mathrm{l}$ pour les placentas et de $1,54 \mathrm{mmol} / \mathrm{l}$ pour les fœtus, la calcémie de $2,1 \mathrm{mmol} / \mathrm{l}$ chez la mère et de 2 à 3,2 mmol/l chez les fœetus observée par Farrell (1984), 
confirme la supériorité de la calcémie fœtale sur la calcémie maternelle comme observé localement. Harter et al. (2015), rapportent aussi une teneur de 0,234 mg/l de calcium dans les liquides fœtaux, ce qui confirme d'avantage nos résultats. Toutefois, les calcémies obtenues localement sont toutes basses et du type hypocalcémiques par rapport à la calcémie de 2,34 $\mathrm{mmol} / \mathrm{l}$, et $1,88 \mathrm{mmol} / \mathrm{l}$ chez les chèvres gestantes observées respectivement par Ouedraogo et al. (2008) et par Harter et al. (2015). Le cas d'hypocalcémie observé localement par rapport aux calcémies exotiques serait dû principalement aux déficits alimentaires, les animaux locaux ne recevant pas généralement des compléments minéraux (Gal et

\section{CONCLUSION}

L'évaluation des teneurs sodiques et calciques, leurs potentialités de transfert dans le CMPF des chèvres gestantes a révélé que la natrémie moyenne est plus concentrée dans le placenta et le fœtus, la gestante demeurant simple source foulante. Par contre, le

\section{REFERENCES BIBLIOGRAPHIQUES}

Barone R., 2001. Anatomie comparée des mammifères domestiques. Splanchnologie II, Tome 4, Vigot-Frères, Paris.

Bell A.W, Greenwood P.I. and Ehrhardt R.A., 2005. Regulation of metabolism and growth during prenatal life. In : Burrin d.G. and Mersmann h.Y.: Biology of Metabolism in Growing Animals, 3, 3-34.

Blain C.J., 2002.Introduction à la nutrition des animaux domestiques. Ed. Technique et Documentation, Paris.

Bossard E. A., Peyraud J. L., Dourmad J.Y., 2009. Effet du bilan électrolytique de la ration sur l'équilibre acido-basique et les performances zootechniques des animaux domestiques à fort niveau de production. INRA. Prod. Anim., 22(2), 117-130.

Boudon A., Khelil-arfa H., Menard J.L., Brunschwing P., Faverdin, 2013. Les besoins en eau d'abreuvement des bovins laitiers: déterminismes physiologiques et quantification. INRA, Prod. Anim., 26(3), 249262.

Farell E.C., 1984 : "Calcium”. Clin. Chim. The C.V.M. Mosby.

Gal O. et Planchanault D., 1993. Utilisation des races caprines exotiques dans les zones chaudes. CIRAD-I.E.M.V.T., Maison-Alfort.
Planchnault, 1993 ;Jarrige et al.,1995 ; Blain, 2002). L'infériorité de la teneur calcique fœtale $(1,54 \mathrm{mmol} / \mathrm{l})$ que nous avons observée localement par rapport à la calcémie de 2 à $3,2 \mathrm{mmol} / /$ observée chez le fœtus exotique par Farrel (1984), serait due non seulement à la faible potentialité de transfert maternel mais aussi à l'intensification des besoins d'ossifications et métaboliques du fœtus. Ce déficit intensifierait l'ostéoporose au cours de la gestation en milieu local et peut même conduire à la dystocie (Payene, 1983). L'état d'hypocalcémie placentaire peut être à la fois dû au transfert maternel insuffisant mais surtout à l'aspiration abondante de calcium par le fœtus pour répondre à ses besoins.

calcium est plus concentré dans le sang fœtal que dans le sang maternel et placentaire. Ces résultats seraient l'expression des sollicitations importantes du fœtus pour les besoins osseux, de croissance et éventuellement pour les liquides fœtaux.

Glles R. ; Anctil M. ; Baguet F. ; Charmantier G., 2006. Physiologie animale. Ed. Deboeck Université, Bruxelles.

Hater C.J, Castagnino D.S., Rivera A.R., Lima L.D, Silva G.O., Mendenca A.N., Bonfin G.F., Liesegang A., Pierre S.N., Teixeira I.A.M.A.A., 2015. Mineral Metabolism in Singleton and Twin-pregnant Dairy Goats. Asian Australas. J.Anim.Sci., 28(1), 37-49.

Jarrige R., Ruckbush Y., Dermarquilly C., Farce M.H., Journet M., 1995. Nutrition des ruminants domestiques. Ingestion et digestion. INRA, Paris.

Kaneko J.J., Cornelius C.E., 1970. Clinical biochemistry of domestic animal. Academic pres, $2^{\text {nd }} \mathrm{Ed}$., New York.

Liesegang A., Risteli J., Vanner M., 2006. The effects of first gestation and lactation on bone metabolism in dairy goats and milk sheep. Bone, 38, 794-802.

Ngoie K., Kasereka S.B., Ndibualonji B.B., Kaputo M., Kaluendi C.M., 2016. Etude des potentialités de transfert des molécules énergétiques dans le complexe maternello-placento -fœtal (CMPF) chez la chèvre en zone tropicale. J. Appl. Biosci., 101, 9653-9660.

Ouedraogo G. A., Barry M., Kanwe B. A., Sawadogo G.J., 2008. Variations des profils métaboliques 
Ngoie et al., J. Appl. Biosci. 2016 Évaluation des teneurs sériques en sodium et en calcium et de leurs potentialités de transfert dans le complexe maternello-placento-fœetal (CMPF) des chèvres en zone tropicale.

lors de gestation à terme et d'avortement chez des chèvres Mossi au Burkina Faso. Revue Méd. Vét, 159 (2) : 112-118.

Payene M.J., 1983. Maladies métaboliques des ruminants domestiques. Ed. Point Vétérinaire, Maison-Alfort.

Pineda M. H., Michael R., Dooley P., 2003. Veterinary endocrinology and reproduction. Fifth edition, Lowa State Press.

Suttle N.F., 2010. Mineral nutrition of liverstock. $4^{\text {th }}$ Edition, CABI, Wallingford, Uk.

Tabatabaei S., 2012. Gestational variations in the biochemical composition of the fetal fluids and maternal blood serum in goats. Comp. Clinical Pathology, 21, 1305-1312. 